\title{
BMJ Open Use of patient-reported outcome measures (PROMs) in clinical diabetes consultations: the DiaPROM randomised controlled pilot trial
}

\author{
Ingvild Hernar (D) , ${ }^{1,2}$ Marit Graue (D) , ${ }^{1}$ David A Richards (D) ,,3 \\ Ragnhild B Strandberg (1) , ${ }^{1}$ Roy Miodini Nilsen (1) , ${ }^{4,5}$ Magne Rekdal, ${ }^{6}$ \\ Karianne Fjeld Løvaas (i) , ${ }^{7}$ Tone V Madsen, ${ }^{7}$ Grethe S Tell (i) , ${ }^{2}$ \\ Anne Haugstvedt (D) ${ }^{1}$
}

To cite: Hernar I, Graue M, Richards DA, et al. Use of patient-reported outcome measures (PROMs) in clinical diabetes consultations: the DiaPROM randomised controlled pilot trial. BMJ Open 2021;11:e042353. doi:10.1136/ bmjopen-2020-042353

- Prepublication history and additional material for this paper are available online. To view these files, please visit the journal online (http://dx.doi. org/10.1136/bmjopen-2020042353).

Received 02 July 2020 Revised 17 December 2020 Accepted 31 March 2021
Check for updates

(c) Author(s) (or their employer(s)) 2021. Re-use permitted under CC BY-NC. No commercial re-use. See rights and permissions. Published by BMJ.

For numbered affiliations see end of article.

Correspondence to Ingvild Hernar;

ingvild.hernar@hvl.no

\section{ABSTRACT}

Objective To pilot test the proposed DiaPROM trial components and address uncertainties associated with conducting a full-scale randomised controlled trial (RCT) to evaluate whether such a trial is feasible.

Design Two-arm pilot RCT.

Participants Adults aged $\geq 18-39$ years, with minimum 1 year type 1 diabetes duration, attending outpatient follow-up. Exclusion criteria were pregnancy, severe cognitive, somatic or psychiatric conditions and impaired vision.

Randomisation and intervention All participants completed electronic Patient-Reported Outcome Measures (PROMs) prior to the annual diabetes consultation. Using computer-generated block-randomisation without blinding, we assigned participants in a 1:1 ratio stratified by sex to receive standard care or an intervention. Physicians reviewed diabetes distress scores (Problem Areas In Diabetes scale) and referred individuals with scores $\geq 30$ or single item(s) $\geq 3$ to minimum two diabetes nurse consultations where reported problems were reviewed and discussed.

Outcomes Recruitment and retention rates; participants perceptions about intervention components. Variance and estimated between-group differences in follow-up scores (Diabetes Distress Scale (DDS), WHO 5-Well-being Index, Perceived Competence for Diabetes Scale and glycaemic control) and DDS correlation with baseline scores, to assist sample size calculations.

Results We randomised 80 participants to the control or intervention arm (one participant was later excluded). 23/39 intervention arm participants qualified for additional consultations and 17 attended. 67/79 attended the 12-month follow-up (15.2\% attrition); 5/17 referred to additional consultations were lost to follow-up $(29.4 \%$ attrition). Participants reported PROMs as relevant (84.6\%) and acceptable (97.4\%) but rated the usefulness of consultations as moderate to low. Baseline mean \pm SD DDS score was 2.1 \pm 0.69 ; DDS SD was 0.71 (95\% Cl: 0.60 to 0.86 ) at follow-up; correlation between baseline and follow-up DDS scores was 0.8 (95\% Cl: 0.7 to 0.9 ).

Conclusions The pilot trial revealed need for intervention modifications ahead of a full-scale trial to evaluate use of
Strengths and limitations of this study

- This pilot trial systematically addressed procedural uncertainties associated with designing a largescale randomised controlled trial.

- The pilot trial allowed us to test the feasibility of Patient-Reported Outcome Measures as dialogue support tools in clinical diabetes consultations.

- Well-known, validated tools for measuring the primary and secondary outcomes facilitated comparison with other studies.

- One of the limitations was that we did not specifically predefine retention and attrition criteria for trial progression.

- Logistical challenges concerning cancelled appointments and non-attendance contributed to difficulties implementing the intervention as designed.

PROMs in diabetes consultations. Specifically, participant acceptability and intervention implementation need further investigation.

\section{BACKGROUND}

Type 1 diabetes (T1D) is a chronic, autoimmune disease which requires lifelong insulin therapy. ${ }^{1}$ Self-management of T1D, the cornerstone of diabetes care, can be described as a 24-hour activity with a constant need to make complex medical decisions and perform challenging diabetes selfmanagement tasks. ${ }^{2}$ During emerging and young adulthood, multiple transitions and developmental stressors can trigger additional self-management difficulties. ${ }^{3}$ Despite advancements in glucose monitoring, insulin therapy and insulin delivery devices, the burden of living with T1D remains a significant challenge. ${ }^{45}$ Only $20 \%-30 \%$ of young adults with T1D achieve recommended glycaemic treatment goals. ${ }^{6-9}$ Poor general 
well-being and emotional distress are known barriers for self-management, and performing behavioural adjustments necessary to promote effective self-management can be challenging. ${ }^{10}$ In addition, individual efforts to achieve beneficial outcomes may not produce desired results. ${ }^{11}$ Diabetes guidelines recommend routine assessment of psychological, emotional and psychosocial factors that impact personal ability to self-manage, like diabetes distress. ${ }^{2}{ }^{12}$ Nevertheless, recent studies indicate that biomedical outcomes receive disproportionate attention in routine follow-up compared with what people with diabetes find important, such as psychosocial aspects. ${ }^{13} 14$

The construct diabetes distress refers to specific negative emotional experiences related to the challenges of living with and managing diabetes and the risk of acute and long-term complications. ${ }^{10} 1516$ Diabetes distress is regarded as an expected reaction first of all impacting on well-being. ${ }^{17}$ In T1D studies, regimen distress, fear of hypoglycaemia and complications, feeling overwhelmed and worrying about the future is most commonly reported. ${ }^{18}$ Furthermore, diabetes distress is more prevalent among younger than older adults ${ }^{3}$ and associated with problematic self-management behaviours related to insulin treatment, glucose monitoring and unsatisfactory glycaemic control. ${ }^{19-22}$ Regimen distress appears to drive these associations. ${ }^{18}$ However, distress may also occur in individuals who reach recommended treatment goals. ${ }^{23}$ Left untreated, mild cases may develop into severe and even chronic distress. ${ }^{24}$ In addition, diabetes distress is found to be a risk factor for symptoms of depression. ${ }^{25}$ This highlights the importance of addressing diabetes distress in routine diabetes care. ${ }^{17} 26$

Patient-reported outcome measures (PROMs) are self-report questionnaires measuring patients' subjective appraisal of a condition, treatment or other healthrelated outcomes. ${ }^{27}$ In clinical consultations, PROMs can be used to increase attention to individual needs, values and preferences. By using PROMs regularly, healthcare providers can screen for self-reported health outcomes, track progress over time and enhance communication with patients. ${ }^{27-30}$ Prior to implementation in clinical care settings, studies are needed to evaluate the feasibility, acceptability and effect of using PROMs in routine consultations. We used the Medical Research Council's (MRC) framework for developing and evaluating complex interventions for guidance. ${ }^{31} 32$ Accordingly, we developed the Diabetes Patient-Reported Outcome Measures (DiaPROM) trial (ClinicalTrials.gov ID: NCT03471104). The overarching aim was to develop, test and evaluate a structured empowerment-based intervention using PROMs regarding diabetes distress as dialogue support in diabetes consultations among adults with T1D. ${ }^{33}$ Furthermore, we hypothesise that the DiaPROM intervention will reduce diabetes distress and improve overall well-being, perceived competence for diabetes management and glycaemic control. First, we conducted a feasibility study to test the technical and practical feasibility and acceptability of capturing PROMs on a touchscreen computer in an outpatient clinic. ${ }^{34}$ Then, we conducted the present pilot trial to test all the components of an upcoming fully powered randomised controlled trial (RCT), to determine if such a trial is feasible and appropriate. Here we report the results of the pilot trial using the Consolidated Standards of Reporting Trials 2010 statement: extension to randomised pilot and feasibility trials. ${ }^{35}$ Findings from qualitative work undertaken alongside the pilot trial are reported elsewhere. ${ }^{3637}$

\section{METHODS}

\section{Aim}

To pilot test the proposed DiaPROM trial components and address uncertainties associated with conducting a full-scale RCT in order to evaluate whether the trial methods and the intervention are feasible. The pilot trial objectives were thus to:

1. Evaluate the recruitment procedures, randomisation procedure and attrition rates.

2. Evaluate the acceptability, appropriateness and implementation of the intervention components.

3. Estimate variance and between-group differences in participant outcomes (diabetes distress; general wellbeing; perceived diabetes competence and glycaemic control) following intervention or standard care, and correlation between participants' diabetes distress scores at baseline and 12 months, in order to assist future sample size calculations.

\section{Design}

The study was designed as a single-centre two-arm pilot RCT.

\section{Setting and participants}

In Norway, people with T1D are followed up at hospital clinics. We conducted the pilot trial at a university hospital endocrinology outpatient clinic where approximately $80 \%$ of the patients with diabetes have T1D. Eligible participants aged $\geq 18-39$ years with T1D duration for at least 1 year were identified using the clinic's attendance list. We sent invitation letters with consent forms by mail 10-14 days prior to the patients' annual diabetes consultations. Informed by pilot trial sample size guidance and the diabetes distress proportions documented in our feasibility study, ${ }^{34}{ }^{38}$ we aimed to recruit 80 participants, 40 in each arm. Using information from the electronic patient records (EPR), we applied the following exclusion criteria: ongoing pregnancy, severe cognitive deficiency, severe somatic comorbidity (eg, end-stage renal disease, severe heart failure, severe cancer), major psychiatric diagnosis (eg, severe depression or bipolar disorder, schizophrenia) and/or impaired vision.

\section{Pilot trial intervention}

We have described the intervention in detail in our protocol paper. ${ }^{33}$ Briefly, DIPS, eHealth systems supplier to Norwegian hospitals, developed the technical application 
for capturing and transferring electronic PROMs to the diabetes-specific EPR. ${ }^{39}$ We asked all participants to arrive 15 min early to complete PROMs on a stationary touchscreen computer located in the outpatient clinic's waiting area prior to two annual diabetes consultations (baseline and 12 months). While completing PROMs, participants received an individual four-character code which was used to download the PROMs to the EPR. The length of the annual consultations was increased from 30 to $45 \mathrm{~min}$. Furthermore, we used the 20-item Problem Areas In Diabetes (PAID) scale to assess diabetes distress. ${ }^{40-42}$ PAID items are rated on a 5-point Likert-like scale (0, 'not a problem' to 4 , 'serious problem'), and an overall diabetes distress score of $0-100$ is calculated, with higher scores indicating greater distress. A score $\geq 40$ suggests serious diabetes distress. ${ }^{104}$ The PAID is widely used, and the Norwegian version is available in the diabetes-specific EPR. ${ }^{43}$

We developed a manual to guide the physicians to download PROMs and review and discuss PAID scores with intervention arm participants, and to identify moderate and serious distress, specifically PAID total score $\geq 30$ or at least one item scored 3 or 4 . Next, the physicians were to offer individuals with such scores a minimum of two 30-min diabetes specialist nurse consultations; the first within 4 weeks after randomisation and the second within a further 3 months. We also developed a communication manual where we guided the nurses to review baseline PAID and discuss reported problem areas with the participants using person-centred, empowerment-based communication skills; 'asking open questions', 'active listening', 'responding', 'summing up' and 'agreeing on goals and actions to take'. In addition, we requested the nurses to record problem areas discussed, goals, action strategies and plans in the EPR. In the second consultations, we asked the nurses and participants to discuss the problem areas, goals and actions and to decide whether to continue with consultations (optional number) until the next annual physician consultation. Intervention arm participants with lower PAID scores received follow-up according to standard clinical protocols after the brief review of their PAID scores with the physicians. Control arm participants, whose scores were inaccessible to the clinicians in the EPR, received 'care as usual'.

\section{Outcomes}

\section{Recruitment}

We recorded the number of individuals invited, number of people attending consultations and number of people who consented to participate in the pilot trial. At baseline, we observed if eligible participants started the PROM sessions by themselves and provided a friendly reminder or assistance to those who did not. At 12 months, we performed similar observations and guidance.

\section{Sample characteristics}

Sociodemographic and diabetes-related information was gathered from the participants' EPR: age, sex, ethnic origin, diabetes duration, diabetes long-term complications, comorbidities, body mass index, glycosylated haemoglobin (Haemoglobin $\mathrm{A}_{1 \mathrm{c}}\left(\mathrm{HbA}_{1 \mathrm{c}}\right)$ ) level, number of self-reported symptomatic hypoglycaemic events in the previous month, history of hypoglycaemia requiring assistance and hospitalisation due to ketoacidosis and insulin injection device. We also obtained self-report data on current type of glucose monitoring device, daily glucose measurement count, first language, educational level, cohabitation status and work affiliation. In addition, we received ethical approval to record age, sex and $\mathrm{HbA}_{1 \mathrm{c}}$ of eligible participants who declined participation.

\section{Primary outcome measure}

To avoid using the same questionnaire for diabetes distress assessment as an element of the intervention and as an outcome measure, we chose the Diabetes Distress Scale (DDS) as our primary outcome. ${ }^{43}$ The 17 -item DDS measures diabetes-specific problems rated on a 6-point Likert-like scale ( 1 , 'no problem' to 6 , 'serious problem'). ${ }^{44}$ The scale yields an overall diabetes distress score and four subscales: emotional burden (five items; eg, 'Feeling that diabetes controls my life'), physician-related distress (four items; eg, 'Feeling that my doctor doesn't take my concerns seriously enough'), regimen-related distress (five items; eg, 'Feeling that I am not testing my blood sugars frequently enough') and diabetes-related interpersonal distress (three items; eg, 'Feeling that friends or family are not supportive enough of self-care efforts'). Item scores are averaged to form a total and subscale scores from 1 to 6 , with higher values indicating greater distress. ${ }^{45}$ Scores are then categorised as little or no distress $(<2.0)$, moderate distress (2.0-2.9) and high distress ( $\geq 3.0)$. Moderate and high distress is considered clinically relevant. ${ }^{45}$

\section{Secondary outcomes measures}

We used the WHO 5-Well-being Index (WHO-5) 5-item measure of current general well-being. ${ }^{46}$ Items are scored on a 6-point Likert-like scale ( 0 , 'at no time' to 5 , 'all the time'). A $0-100$ score is calculated and scores $<50$ suggest impaired well-being, while $\leq 28$ indicate likely depression. ${ }^{46} 47$ A 10-point change is considered clinically relevant. ${ }^{46}$ The measure is reported to be psychometrically sound, acceptable and suitable for diabetes outpatient settings. ${ }^{48-50}$ The 4-item Perceived Competence for Diabetes Scale (PCDS) assesses the degree to which people with diabetes feel they can manage daily aspects of diabetes care (1, 'strongly disagree' to 7 , 'strongly agree').$^{51}$ Item scores are averaged to form a score. Finally, we obtained information about glycaemic control from routinely performed blood samples measuring $\mathrm{HbA}_{1 \mathrm{c}}(\mathrm{mmol} / \mathrm{mol})$ recorded in the EPR.

\section{Experiences with the pilot trial intervention}

After each annual consultation, participants were asked to complete a paper questionnaire, which included the DDS (primary outcome measure) and questions about experiences with and perceptions about the pilot trial 
components. We asked all participants PROMs acceptability questions (five response options from 'not at all' to 'very large degree'): relevance, number of items and willingness for annual completion. In addition, we asked about preferred completion method (electronic or paper). Intervention arm participants were also asked about PAID use and consultation usefulness. Finally, we reviewed the nurses' EPR notes for intervention arm participants referred to additional follow-up, to evaluate intervention consultation fidelity (per-protocol).

\section{Randomisation}

We randomised participants in a 1:1 ratio to an intervention or control arm using computer-generated block-randomisation at the patient level, developed and administered by DIPS. ${ }^{39}$ The computerised allocation took place when the physicians downloaded PROMs to the EPR. Group allocation information appeared on the computer screen, and the physicians told the participants. Furthermore, we stratified by sex to ensure equal numbers (20) of male and female participants in each arm. Due to the nature of the intervention, blinding of group allocation to participants, healthcare providers and research personnel was not possible.

\section{Analyses}

All analyses were carried out using Stata SE 16 for Windows. ${ }^{52}$ At each timepoint, we estimated means, SD and 95\% CI of SDs of outcome measures for both groups. To examine within and between-group variation of paired differences in outcome measures from baseline to 12-month follow-up, we estimated means and SDs, and means and 95\% CIs, respectively. Using Spearman's correlation coefficient, we estimated correlation with 95\% CI between participants' primary outcome measure scores at baseline and 12 months. The primary outcome measure SD, 95\% CI of SD and correlation coefficient was used to assist in full trial sample size calculations. In all analyses, we computed missing items using person-mean substitution if at least $50 \%$ of the items per scale were completed. ${ }^{53} 54$

\section{Patient and public involvement}

In the protocol paper, ${ }^{33}$ we have provided a detailed description of health service user involvement based on the Guidance for Reporting Involvement of Patients and the Public 2 (GRIPP2) short form. ${ }^{55}$

\section{RESULTS}

\section{Recruitment, randomisation, sample characteristics, and} retention

Between 15 January and 7 May 2018, we assessed 149 patients with T1D for eligibility and randomised 80 participants, $40(50 \%)$ to each trial arm (figure 1). The randomisation procedure yielded two groups with equal distribution of men and women. Baseline characteristics for the total sample and trial arms are presented in table 1. Compared with the included participants, the 22 who declined had longer diabetes duration $(13.7 \pm 7.0$ years (95\% CI: 12.2 to 15.3$)$ vs $18.6 \pm 10.2$ years $(95 \% \mathrm{CI}$ : 14.1 to 23.1)), while there were no differences in gender distribution, age or $\mathrm{HbA}_{1 \mathrm{c}}$ level. Furthermore, 24/40 $(60.0 \%)$ intervention arm participants qualified for additional nurse follow-up (figure 1). One participant was later excluded due to newly discovered language problems. In total, 17/23 (73.9\%) were referred and attended 1-5 consultations $($ mean \pm SD $2.2 \pm 1.1) ; 12 / 17 \quad(70.6 \%)$ attended the per-protocol minimum. After reviewing the nurses' EPR notes, we registered that 28/38 consultations were performed according to the protocol, while 10/38 focused on other aspects than diabetes distress assessed by the PAID. Therefore, a mean of 1.65 (0-2) intervention consultations was conducted, and $9 / 17$ received per-protocol follow-up of minimum two sessions. The 12-month follow-up was performed from 5 December 2018 to 17 June 2019. Twelve participants were lost to follow-up (overall attrition rate $15.2 \%$; intervention arm: 8 (20.5\%); control arm: $4(10 \%))$, but none withdrew consent (figure 1). Furthermore, 5/17 referred to additional nurse consultations were lost to follow-up (attrition rate $29.4 \%$ ).

\section{Acceptability, appropriateness and implementation of the intervention components}

At baseline, 21/79 (26.6\%) participants located the touchscreen computer without guidance, 43 (54.4\%) confirmed they had read the written study information. At 12 months, five participants completed PROMs on paper; four because of a defective touchscreen and one asked for a telephone consultation. Of the remaining 62 participants, we had to remind $30(48.4 \%)$ to complete PROMs. Furthermore, 2/17 participants referred to additional nurse follow-up delayed the first consultation for 4-6 months. The remaining 15/17 were offered the first consultation within 27.0 4.8 (19-35) days after randomisation. However, due to five participants postponing at least once, the consultations were conducted after $42.5 \pm 27.7$ (22-123) days. The second appointments $(n=15)$ were offered after $85.5 \pm 30.6$ (20-133) days and attended by 12 participants after $100.8 \pm 35.3$ (20-153) days.

Total WHO-5, PAID and PCDS completeness was $99.4 \%$ at baseline and $99.2 \%$ at 12 months. When asked about preferred method for completing PROMs in the future, two $(2.6 \%)$ individuals chose paper-completion, whereas $42(54.5 \%)$ opted for in-clinic computerised PROMs and $33(42.9 \%)$ favoured home-based web-completion (online supplemental figure 1). Seventy-five (97.4\%) reported that number of items were acceptable to a large or very large degree, $72(92.3 \%)$ found the items relevant and $66(84.6 \%)$ were willing to complete PROMs annually (online supplemental figure 1).

Among intervention arm participants, 23/39 (59.0\%) and 13/31 (41.9\%) reported PAID items scored $\geq 3$ and/ or a total score $\geq 30$ (moderate to high distress) at baseline and follow-up, respectively (online supplemental table 1 ). 
DiaPROM Pilot Trial CONSORT 2010 Flow Diagram

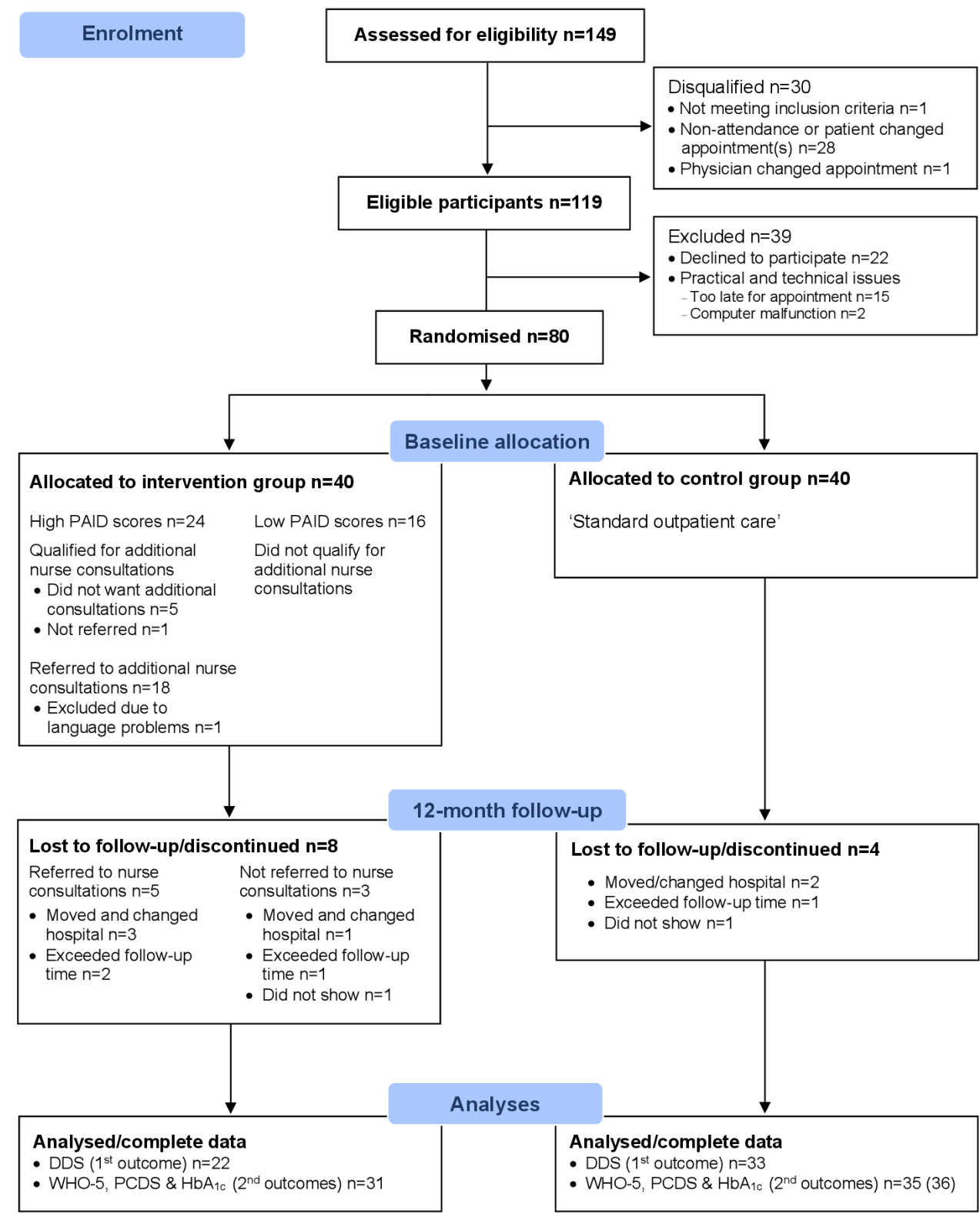

Figure 1 The DiaPROM pilot trial's consolidated standards of reporting trials flow diagram.

(CONSORT, Consolidated Standards of Reporting Trials; DiaPROM, Diabetes Patient-Reported Outcome Measures; DDS,

Diabetes Distress Scale; PAID, Problem Areas In Diabetes; PCDS, Perceived Competence for Diabetes Scale.)

The control arm participants' corresponding proportions were $19 / 40(47.5 \%)$ and $20 / 36(55.6 \%)$. Thirty (76.9\%) intervention group participants reported that the PAID results were discussed at baseline, of which 15 (38.5\%) found it useful to a large or very large degree and 10 (25.6\%) to some degree. At 12 months, 20/24 (83.3\%) reported that PAID was discussed and $11(45.8 \%)$ found it useful to a large or very large degree. Only 10/17 referred to additional follow-up completed all items about PAID use; five found the discussions useful; four reported to have benefitted to a large degree, whereas three had not benefitted at all. In total, $17 / 53(32.1 \%)$ participants stated that completing PROMs had to some degree led to discussions related to diabetes-related challenges which would not otherwise have been discussed (similar in both trial arms). Furthermore, 14 (26.4\%) reported that completing PROMs had been a positive experience, while $24 / 53(45.2 \%)$ found it somewhat positive (similar in both trial arms).

\section{Outcome measures}

In total, $67 / 79(84.8 \%)$ participants responded to all DDS items at baseline and 58/67 (86.6\%) at 12 months (online supplemental table 2). Mean scores and SDs of the outcome measures at baseline and follow-up for each trial arm are reported in tables 2 and 3. At follow-up, the sample's SD of DDS score was 0.71 (95\% CI: 0.60 to 0.86) (table 2). From baseline to follow-up, we observed a 
Table 1 Baseline sociodemographic and clinical characteristics of the DiaPROM pilot trial participants with type 1 diabetes aged $18-40$ years $(n=79)$

\begin{tabular}{|c|c|c|c|}
\hline & \multirow{2}{*}{$\begin{array}{l}\text { All } \\
n=79\end{array}$} & \multicolumn{2}{|l|}{ Groups } \\
\hline & & Intervention, $\mathrm{n}=39$ & Control, $\mathrm{n}=40$ \\
\hline Gender, women & $40(50.6)$ & $20(50.6)$ & $20(50.0)$ \\
\hline Age, years & $27.2 \pm 5.0$ & $27.1 \pm 4.7$ & $27.3 \pm 5.3$ \\
\hline European origin & $79(100.0)$ & $39(100.0)$ & $40(100.0)$ \\
\hline Norwegian first language & $74(93.7)$ & $36(92.3)$ & $38(95.0)$ \\
\hline Living alone & $17(21.5)$ & $4(10.3)$ & $13(32.5)$ \\
\hline University/college education & $37(46.8)$ & $21(53.9)$ & $16(40.0)$ \\
\hline \multicolumn{4}{|l|}{ Work affiliation } \\
\hline Full-time work & $36(45.6)$ & $18(46.2)$ & $18(45.0)$ \\
\hline Part-time work & $5(6.3)$ & $0(0.0)$ & $5(12.5)$ \\
\hline Student & $25(31.7)$ & $15(38.5)$ & $10(25.0)$ \\
\hline Other & $13(16.4)$ & $6(15.3)$ & $7(17.5)$ \\
\hline Diabetes duration, years & $13.7 \pm 7.0$ & $13.6 \pm 6.4$ & $13.9 \pm 7.6$ \\
\hline Long-term complication(s) & $16(20.3)$ & $4(10.3)$ & $12(30.0)$ \\
\hline Retinopathy* & 15 (19.0) & $4(10.3)$ & $11(27.5)$ \\
\hline Nephropathy & $1(1.3)$ & $0(0.0)$ & $1(2.5)$ \\
\hline \multicolumn{4}{|l|}{ Comorbidities/other autoimmune diseases } \\
\hline Thyroid disease & $5(6.3)$ & $2(5.1)$ & $3(7.5)$ \\
\hline Coeliac disease & $8(10.1)$ & $4(10.2)$ & $4(10.0)$ \\
\hline $\mathrm{HbA}_{1 \mathrm{c}}(\mathrm{mmol} / \mathrm{mol}) \dagger$ & $65.4 \pm 14.5$ & $64.8 \pm 13.2$ & $66.0 \pm 15.8$ \\
\hline $\mathrm{HbA}_{1 \mathrm{c}}(\%)$ & $8.1 \pm 1.3$ & $8.1 \pm 1.2$ & $8.2 \pm 1.4$ \\
\hline $\mathrm{HbA}_{1 \mathrm{c}} \leq 53 \mathrm{mmol} / \mathrm{mol}(\leq 7.0 \%) \ddagger$ & $15(19.0)$ & $7(18.0)$ & $8(20.0)$ \\
\hline Body Mass Index (kg/m²) & $24.6 \pm 5.6$ & $24.2 \pm 7.1$ & $25.0 \pm 3.6$ \\
\hline Symptomatic hypos last month & $9.8 \pm 10.5$ & $11.4 \pm 12.6$ & $8.1 \pm 7.5$ \\
\hline Severe hypoglycaemic event ever§ & $34(43.0)$ & $18(46.1)$ & $16(40.0)$ \\
\hline Ketoacidosis (ever hospitalised) & $17(21.5)$ & $7(17.9)$ & $10(25.0)$ \\
\hline Insulin pump & $38(48.1)$ & $20(51.3)$ & $18(45.0)$ \\
\hline Continuous glucose monitoring (CGM) & $22(27.9)$ & $11(28.2)$ & $11(27.5)$ \\
\hline Self-blood glucose monitoring (SBGM) & $53(67.1)$ & $27(69.2)$ & $26(65.0)$ \\
\hline Flash glucose monitoring (FGM) & $2(2.5)$ & $1(2.5)$ & $1(2.5)$ \\
\hline Daily SBGM/FGM川 & 47 (85.5) & $25(89.3)$ & $22(81.5)$ \\
\hline Weekly count SBGM/FGM & $32.8 \pm 22.9$ & $39.4 \pm 25.3$ & $26.7 \pm 18.9$ \\
\hline
\end{tabular}

Data are shown as $n(\%)$ (of patients with valid values) or mean \pm SD.

*Any degree of retinopathy

†Haemoglobin $\mathrm{A}_{1 c}$.

$\ddagger \mathrm{HbA}_{1 \mathrm{c}}$ target achieved.

$\S A$ least one severe hypoglycaemic event with need of assistance (yes).

१Total $\mathrm{n}$ based on participants using SBGM or FGM.

DiaPROM, Diabetes Patient-Reported Outcome Measures.

reduction in DDS overall score by an average of 0.25 (SD: $0.42)$ in the intervention arm but no apparent reduction in the control arm $(0.00, \mathrm{SD}: 0.47)$. The intervention arm's DDS subscale scores were all improved $(-0.14$ to -0.39 , SDs: 0.66 to 0.86 ), while the control arm's changes in subscales scores ranged from -0.07 to 0.09 (SDs: 0.54 to 0.82 ). For other outcome variables (WHO-5, PCDS and $\mathrm{HbA}_{1 c}$ ), only small changes were seen (table 3 ). The correlation coefficient between baseline and follow-up DDS scores was 0.8 (95\% CI: 0.7 to 0.9 ) (online supplemental table 3 ).

In addition, 18/33 (54.5\%) and 11/26 (42.3\%) intervention arm participants reported moderate to high distress measured by the DDS overall score at baseline 


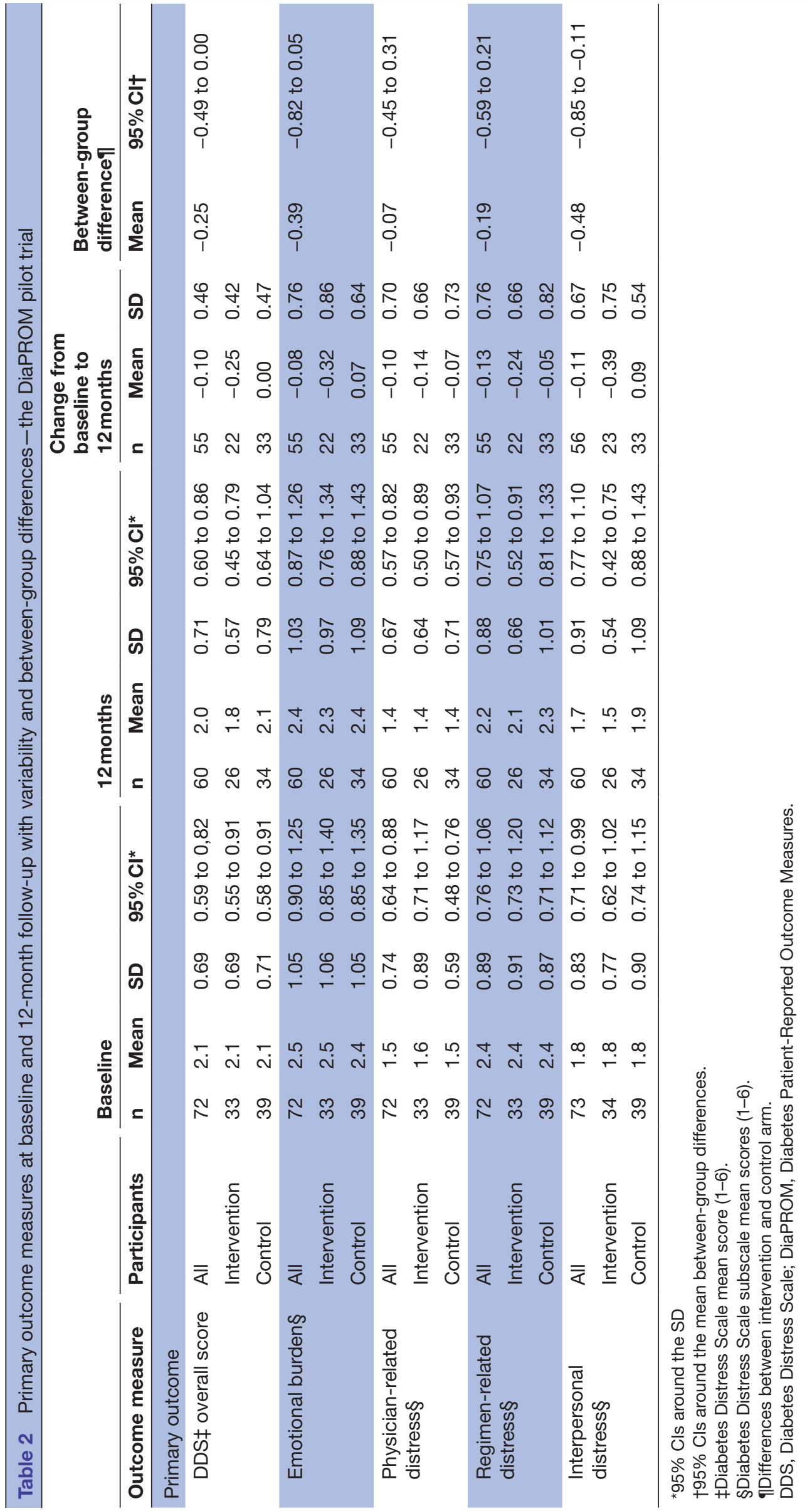




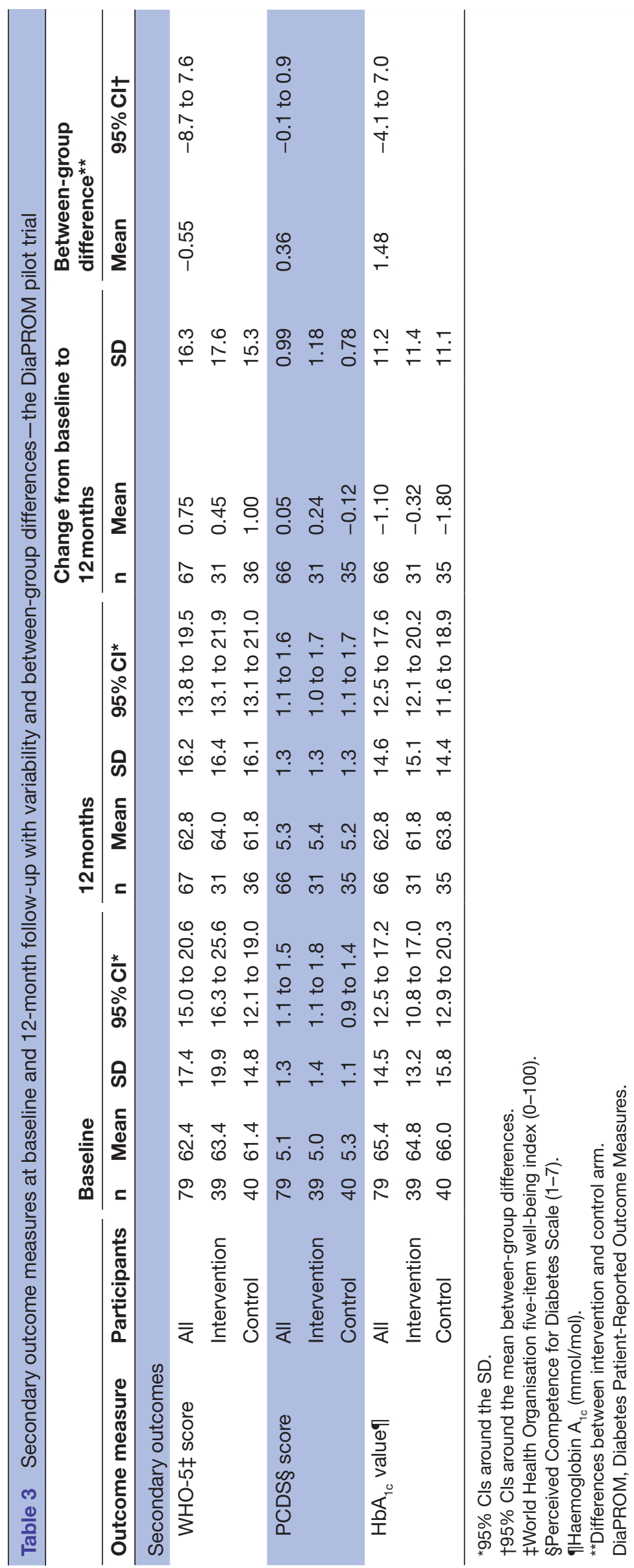

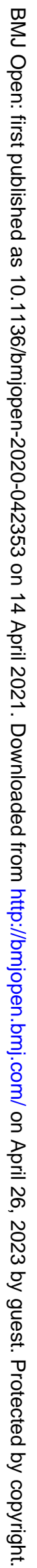


and follow-up, respectively (online supplemental table 4). Corresponding proportions for control arm participants were 15/39 (38.5\%) and 17/34 (50.0\%). Regarding DDS subscales, the percentage of participants reporting moderate to high emotional burden and regimenrelated distress was persistent at $\sim 60 \%$, across groups and timepoints.

\section{DISCUSSION}

In this randomised controlled pilot trial, we found that it was feasible to recruit and randomise young adults with T1D attending routine diabetes consultations to a trial using PAID and communication techniques as dialogue support tools. The participants were positive towards completing PROMs. Furthermore, we were able to retain $67 / 79(84.8 \%)$ participants at 12 months. However, we identified implementation challenges related to the intervention consultations, and 5/17 (29.4\%) participants referred to additional consultations were lost to follow-up at 12 months.

\section{Strengths and limitations}

The pilot trial's key strengths were that it systematically addressed uncertainties associated with designing a largescale RCT. Moreover, well-known, validated tools for measuring primary and secondary outcomes allowed for comparison with other studies. The results inform technical and practical issues of conducting a full-scale trial. Similar to our feasibility study, ${ }^{34}$ findings suggest that completing electronic PROMs was generally accepted and technically feasible. We were able to recruit and randomise 80 participants over 15 weeks. However, one fundamental limitation was that the 12-month follow-up lasted nearly twice as long (28weeks), mainly caused by cancelled appointments, non-attendance and loss to follow-up. Another limitation was not having predefined criteria for retention and attrition progression rates. However, this is not yet common. ${ }^{56}$ Furthermore, complete follow-up was not achieved, and attrition differed by trial arm: $10 \%$ in the control arm, $20 \%$ in the intervention arm and $29 \%$ among those who were referred to additional follow-up. Systematic differences between completers and drop-outs may have introduced attrition bias. ${ }^{57}$ However, clinic nonattendance is not uncommon among young adults with T1D and has been linked to difficulties communicating with the services, conflicting schedules, low perceived value of attendance and challenges with developing relationships. ${ }^{589}$ The retention, implementation and acceptability issues are further explored in qualitative interviews. In summary, these issues will impact power calculations by increasing the target sample needed, in addition to affecting intervention implementation and the duration of a full-scale RCT.

Generalisability and transferability to other settings and populations may be limited due to our use of electronic technologies for completing PROMs, our choice of only including young adults with T1D, and that the Norwegian health insurance system differs from other countries. Finally, although PAID scores were not accessible in the control arm participants' EPRs, we cannot rule out contamination. Since all participants completed PROMs in the same manner, control arm participants' consultations may have been influenced by individual responses and thoughts about the questionnaires. Moreover, consulting styles within a service probably differs between clinicians. For ethical reasons, we could not instruct the physicians to avoid discussing diabetes distress in the control arm if participants requested it.

\section{Implications and future research}

Using current pilot trial data and a conservative DDS SD estimate to calculate the minimal clinically important difference $(0.5 \times \mathrm{SD}),{ }^{60}$ and assuming that $\mathrm{SD}(0.71)$ is equal for each trial arm in a full-scale, single-site RCT, we estimate at least 107 participants will be required per arm to provide $90 \%$ power based on a two-sided $5 \%$ significance level. The calculation was based on the formula of a two-sample t-test for difference post-intervention and allowed for $25 \%$ attrition.

Since $10 \%$ of the participants did not complete the paper-based measures, and there was considerably more missing DDS items than other PROM items, we will strive for capturing all future data electronically. Furthermore, only a minority of participants approached the touchscreen computer by themselves. Therefore, we will continue with in-clinic guidance and e-mail or SMS reminders to support data collection. A web-based PROMs platform, recently available in Norway, will possibly enable more complete data collections in future studies. Moreover, we observed that $36(45.6 \%)$ participants had not read the study information prior to coming to the clinic but still consented. The drop-out rates and other findings suggest that consultations were not considered useful, adequate or appropriate by the participants. This could in part be explained by protocol inflexibility and/or the waiting time between PROMs completion and additional consultations. In addition, we may not have provided sufficient detailed information about the nature of the intervention components, especially the additional follow-up. Also, this key intervention component may not have fitted the participants' personal beliefs, preferences, capabilities and/or life circumstances. ${ }^{61}$ We may also have underestimated the contribution of the baseline review of scores and discussions between intervention arm participants and physicians. Furthermore, our criteria for offering additional follow-up may have led to overinclusion of cases but we must also consider barriers to clinic attendance and dissatisfaction with the follow-up. ${ }^{1458}$ Another aspect which requires consideration, is that simply answering questions for assessment purposes, such as PROMs, may affect research participants by stimulating new thinking about problem areas or behaviours, which then may lead to action-taking. ${ }^{62}$ This question-behaviour effect makes it even more difficult to evaluate complex interventions. 
Diabetes distress scores were similar to previous studies. ${ }^{2021} 63$ Approximately half of the participants reported moderate to serious distress, which supports statements that diabetes distress is common and worthy of individual attention in diabetes care. ${ }^{412} 15$ Although the pilot trial was not powered for inferential statements, the observed between-group differences in DDS scores suggest promising effects of assessing and addressing diabetes distress. Compared with lack of assessment and follow-up, education-based or emotion-focused interventions targeting diabetes distress in adults with T1D have been found clinically effective. ${ }^{246465}$ In the pilot trial, we focused on real-life clinical consultations. Hence, the clinicians meet individuals with different needs which may entail applying either education-based or emotionfocused interventions or both, depending on individual diabetes distress foci, discussions with each individual and clinical experience. Personalising diabetes care by addressing diabetes distress systematically, may increase healthcare providers' attentiveness towards the individual experiences of living with diabetes.

Implementation fidelity and difficulties in delivering the intervention as designed appeared challenging for the clinic. One aspect was providing the consultations within the specified timeframe. Recommendations of 2-week to 1-month intervals between consultations ${ }^{23} 66$ may be difficult to achieve within regular working hours unless telephone or digital communication are used. ${ }^{67}$ The observed lack of intervention fidelity, for example, not reviewing the PAID during annual consultations, may be partly explained by low sense of project ownership from the clinicians. This highlights the importance of organisational incentives, management facilitation of new intervention initiatives and possibly cultural aspects in this setting. Our efforts to encourage intervention fidelity by providing information, developing manuals and arranging meetings and training for the clinicians may not have been sufficient. Consequently, we must seek to further identify key contextual, organisational and behavioural factors and mechanisms of impact. The pilot trial results show that we must involve health service users and clinicians in further development of the intervention and undertake more feasibility work with process evaluations to inform the design of a full-scale trial. ${ }^{68}$

\section{CONCLUSIONS}

Results from this randomised controlled pilot trial show that it is feasible to recruit and randomise young adults with T1D attending an outpatient clinic to a study using electronically captured PROMs to assess diabetes distress. However, the intervention was not provided as planned. Low perceived usefulness and high attrition rate among intervention arm participants also suggest low acceptability or overinclusion. The pilot trial revealed problems with design and deliverability and highlighted the need for several intervention modifications before initiating a full-scale evaluation of using electronic PROMs in diabetes consultations.

Author affiliations

${ }^{1}$ Department of Health and Caring Sciences, Western Norway University of Applied Sciences, Bergen, Norway

${ }^{2}$ Department of Global Public Health and Primary Care, University of Bergen, Bergen, Norway

${ }^{3}$ Institute for Health Research, College of Medicine and Health, University of Exeter, Exeter, UK

${ }^{4}$ Faculty of Health and Social Sciences, Western Norway University of Applied Sciences, Bergen, Norway

${ }^{5}$ Department of Research and Development, Haukeland University Hospital, Bergen, Norway

${ }^{6}$ Dips AS, Bergen, Norway

${ }^{7}$ Norwegian Diabetes Register for Adults, Norwegian Organisation for Quality Improvement of Laboratory Examinations (Noklus), Haraldsplass Deaconess Hospital, Bergen, Norway

Twitter Ingvild Hernar @|Hernar and Anne Haugstvedt @Anne Haugstvedt

Acknowledgements We wish to thank the study participants for their invaluable contribution, and the clinicians at the outpatient clinic for their involvement. We also wish to thank the entire DiaPROM project group for their input and support. With special thanks to the user representatives, Silje S Lie and Inger Myrtvedt and Bojan Nikolic at Dips.

Contributors All authors have contributed in accordance with the criteria for authorship. $A H$ and $M G$ applied for funding. $A H, I H, M G$ and $R B S$ designed the study with involvement of GST, DAR, RMN, MR, KFL and TVM. IH wrote the first draft of this manuscript. AH, MG, DAR, RBS, RMN and GST gave substantial contributions to the interpretation of data and revised the manuscript critically. All other authors have edited and critically reviewed the manuscript. Finally, all authors read and approved the final version of the paper.

Funding The study was funded by the Norwegian Nurses Association (NNA), the Western Norway University of Applied Sciences (HVL), the Norwegian Diabetes Association (NDA) and the Norwegian Diabetes Register for Adults (NDR-A). The first two funded a postdoctoral position and a PhD position, respectively. The last two contributed with running funds. David Richards receives support from the UK National Institute for Health Research (NIHR) Applied Research Collaboration (ARC) South West Peninsula (NIHR200167). The views expressed are those of the authors and not necessarily those of the NNA, HVL, NDA, NDR-A, National Health Service, NIHR or the Department of Health and Social Care.

Competing interests None declared.

Patient consent for publication Not required.

Ethics approval The study was approved by the Regional Committee for Medical and Health Research Ethics (reference number 2017/1506/REK vest) and was undertaken in accordance with the Declaration of Helsinki. All study data have been stored on a secure research server at Haukeland University Hospital, the responsible research institution. Participation was voluntary and participants gave informed written consent. Participants were free to withdraw their consent at any time without explanation, and withdrawal would not affect their further follow-up at the outpatient clinic.

Provenance and peer review Not commissioned; externally peer reviewed.

Data availability statement Data are available upon reasonable request.

Supplemental material This content has been supplied by the author(s). It has not been vetted by BMJ Publishing Group Limited (BMJ) and may not have been peer-reviewed. Any opinions or recommendations discussed are solely those of the author(s) and are not endorsed by BMJ. BMJ disclaims all liability and responsibility arising from any reliance placed on the content. Where the content includes any translated material, BMJ does not warrant the accuracy and reliability of the translations (including but not limited to local regulations, clinical guidelines, terminology, drug names and drug dosages), and is not responsible for any error and/or omissions arising from translation and adaptation or otherwise.

Open access This is an open access article distributed in accordance with the Creative Commons Attribution Non Commercial (CC BY-NC 4.0) license, which permits others to distribute, remix, adapt, build upon this work non-commercially, and license their derivative works on different terms, provided the original work is 
properly cited, appropriate credit is given, any changes made indicated, and the use is non-commercial. See: http://creativecommons.org/licenses/by-nc/4.0/.

\section{ORCID iDs}

Ingvild Hernar http://orcid.org/0000-0001-7185-6234

Marit Graue http://orcid.org/0000-0002-3149-6227

David A Richards http://orcid.org/0000-0002-8821-5027

Ragnhild B Strandberg http://orcid.org/0000-0003-0256-438X

Roy Miodini Nilsen http://orcid.org/0000-0002-0228-1550

Karianne Fjeld Løvaas http://orcid.org/0000-0002-1658-0973

Grethe S Tell http://orcid.org/0000-0003-1386-1638

Anne Haugstvedt http://orcid.org/0000-0002-9742-295X

\section{REFERENCES}

1 Atkinson MA, Eisenbarth GS, Michels AW. Type 1 diabetes. Lancet 2014:383:69-82.

2 American Diabetes Association. 5. Facilitating Behavior Change and Well-being to Improve Health Outcomes: Standards of Medical Care in Diabetes-2020. Diabetes Care 2020;43:S48-65.

3 Wentzell K, Vessey JA, Laffel LMB. How do the challenges of emerging adulthood inform our understanding of diabetes distress? an integrative review. Curr Diab Rep 2020;20:21.

4 Jones A, Vallis M, Pouwer F. If it does not significantly change $\mathrm{HbA} 1 \mathrm{c}$ levels why should we waste time on it? a plea for the prioritization of psychological well-being in people with diabetes. Diabet Med 2015;32:155-63.

5 Kubiak T, Priesterroth L, Barnard-Kelly KD. Psychosocial aspects of diabetes technology. Diabet Med 2020;37:448-54.

6 Carlsen S, Skrivarhaug T, Thue G, et al. Glycemic control and complications in patients with type 1 diabetes - a registry-based longitudinal study of adolescents and young adults. Pediatr Diabetes 2017:18:188-95.

7 Cooper JG, Bakke Å, Dalen I, et al. Factors associated with glycaemic control in adults with type 1 diabetes: a registry-based analysis including 7601 individuals from 34 centres in Norway. Diabet Med 2020;37:828-37.

8 McKnight JA, Wild SH, Lamb MJE, et al. Glycaemic control of Type 1 diabetes in clinical practice early in the 21st century: an international comparison. Diabet Med 2015;32:1036-50.

9 Foster NC, Beck RW, Miller KM, et al. State of type 1 diabetes management and outcomes from the T1D exchange in 2016-2018. Diabetes Technol Ther 2019;21:66-72.

10 Snoek FJ, Bremmer MA, Hermanns N. Constructs of depression and distress in diabetes: time for an appraisal. Lancet Diabetes Endocrinol 2015;3:450-60.

11 Rubin RR. Counselling and psychoterapy in diabetes mellitus. In: Snoek FJ, Skinner TC, eds. Psychology in diabetes care. 2nd ed. Chichester: Wiley, 2005: 171-93.

12 Young-Hyman D, de Groot M, Hill-Briggs F, et al. Psychosocial care for people with diabetes: a position statement of the American diabetes association. Diabetes Care 2016;39:2126-40.

13 Litterbach E, Holmes-Truscott E, Pouwer F, et al. 'I wish my health professionals understood that it's not just all about your $\mathrm{HbA}_{1}$ !'. Qualitative responses from the second Diabetes MILES - Australia (MILES-2) study. Diabet Med 2020;37:971-81.

14 Iversen E, Kolltveit B-CH, Hernar I, et al. Transition from paediatric to adult care: a qualitative study of the experiences of young adults with type 1 diabetes. Scand J Caring Sci 2019;33:723-30.

15 Skinner TC, Joensen L, Parkin T. Twenty-Five years of diabetes distress research. Diabet Med 2020;37:393-400.

16 Dennick K, Sturt J, Speight J. What is diabetes distress and how can we measure it? A narrative review and conceptual model. J Diabetes Complications 2017;31:898-911.

17 Fisher L, Gonzalez JS, Polonsky WH. The confusing tale of depression and distress in patients with diabetes: a call for greater clarity and precision. Diabet Med 2014;31:764-72.

18 Sturt J, Dennick K, Due-Christensen M, et al. The detection and management of diabetes distress in people with type 1 diabetes. Curr Diab Rep 2015;15:101.

19 Strandberg RB, Graue M, Wentzel-Larsen T, et al. Longitudinal relationship between diabetes-specific emotional distress and follow-up HbA1c in adults with type 1 diabetes mellitus. Diabet Med 2015;32:1304-10.

20 Fisher L, Hessler D, Polonsky W, et al. Diabetes distress in adults with type 1 diabetes: prevalence, incidence and change over time. $J$ Diabetes Complications 2016;30:1123-8.
21 Hessler DM, Fisher L, Polonsky WH, et al. Diabetes distress is linked with worsening diabetes management over time in adults with type 1 diabetes. Diabet Med 2017;34:1228-34.

22 Hagger V, Hendrieckx C, Cameron F, et al. Diabetes distress is more strongly associated with $\mathrm{HbA} 1 \mathrm{c}$ than depressive symptoms in adolescents with type 1 diabetes: results from diabetes miles YouthAustralia. Pediatr Diabetes 2018;19:840-7.

23 Fisher L, Polonsky WH, Hessler D. Addressing diabetes distress in clinical care: a practical guide. Diabet Med 2019;36:803-12.

24 Hessler D, Fisher L, Polonsky W, et al. There is value in treating elevated levels of diabetes distress: the clinical impact of targeted interventions in adults with type 1 diabetes. Diabet Med 2020;37:71-4.

25 Ehrmann D, Kulzer B, Haak T, et al. Longitudinal relationship of diabetes-related distress and depressive symptoms: analysing incidence and persistence. Diabet Med 2015;32:1264-71.

26 Snoek FJ, Kersch NYA, Eldrup E, et al. Monitoring of Individual Needs in Diabetes (MIND)-2: follow-up data from the cross-national Diabetes Attitudes, Wishes, and Needs (DAWN) MIND study. Diabetes Care 2012;35:2128-32.

27 Greenhalgh J, Dalkin S, Gooding K. Functionality and feedback: a realist synthesis of the collation, interpretation and utilisation of patient-reported outcome measures data to improve patient care. health services and delivery research. 318. Southampton (UK: NIHR Journals Library (Health Services and Delivery Research, No. 5.2.), 2017.

28 International Society for Quality of Life Research. What are your goals for collecting PROs in your clinical practice and what resources are available? Which key barriers require attention? In: Aaronson N, Elliott T, Greenhalgh J, eds. User's guide to implementing patient-reported outcomes assessment in clinical practice. 2nd ed. Milwaukee, WI, US: International Society for Qualiy of Life Research, 2015: 4-6. https://www.isoqol.org/wp-content/uploads/2019/09/ 2015UsersGuide-Version2.pdf

29 International Society for Qualiy of Life Research. What are your goals for collecting PROs in your clinical practice and what resources are available? Which key barriers require attention? In: Chan E, Edwards T, Haywood K, eds. Implementing patient-reported outcome measures in clinical practice: a companion guide to the ISOQOL user's guide. 1st ed. Milwaukee, WI, US: International Society for Quality of Life Research, 2018: 2-4. https://www.isoqol. org/wp-content/uploads/2019/09/ISOQOL-Companion-GuideFINAL.pdf

30 Porter I, Gonçalves-Bradley D, Ricci-Cabello I, et al. Framework and guidance for implementing patient-reported outcomes in clinical practice: evidence, challenges and opportunities. J Comp Eff Res 2016;5:507-19.

31 Craig P, Dieppe P, Macintyre S, et al. Developing and evaluating complex interventions: the new medical Research Council guidance. BMJ 2008;337:a1655.

32 Craig P, Petticrew M. Developing and evaluating complex interventions: reflections on the 2008 MRC guidance. Int J Nurs Stud 2013;50:585-7.

33 Haugstvedt A, Hernar I, Strandberg RB, et al. Use of patient-reported outcome measures (PROMs) in clinical diabetes consultations: study protocol for the DiaPROM randomised controlled trial pilot study. BMJ Open 2019;9:e024008.

34 Hernar I, Graue M, Richards D, et al. Electronic capturing of patientreported outcome measures on a touchscreen computer in clinical diabetes practice (the DiaPROM trial): a feasibility study. Pilot Feasibility Stud 2019;5:29.

35 Eldridge SM, Chan CL, Campbell MJ, et al. Consort 2010 statement: extension to randomised pilot and feasibility trials. BMJ 2016;355:i5239.

36 Haugstvedt A, Hernar I, Graue M, et al. Nurses' and physicians' experiences with diabetes consultations and the use of dialogue tools in the DiaPROM pilot trial: A qualitative study. Diabet Med 2020:e14419.

37 Hernar I, Graue M, Strandberg RB, et al. Young adults with type 1 diabetes and their experiences with diabetes follow-up and participation in the DiaPROM pilot trial: a qualitative study. Diabet Med 2021:e14535.

38 Thabane L, Ma J, Chu R, et al. A tutorial on pilot studies: the what, why and how. BMC Med Res Methodol 2010;10:1.

39 DIPS AS. Supplier of eHealth systems to Norwegian hospitals, 2020. Available: https://www.dips.com/uk

40 Polonsky WH, Anderson BJ, Lohrer PA, et al. Assessment of diabetes-related distress. Diabetes Care 1995;18:754-60.

41 Welch GW, Jacobson AM, Polonsky WH. The problem areas in diabetes scale. An evaluation of its clinical utility. Diabetes Care 1997;20:760-6. 
42 Welch G, Weinger K, Anderson B, et al. Responsiveness of the problem areas in diabetes (paid) questionnaire. Diabet Med 2003;20:69-72.

43 Graue M, Haugstvedt A, Wentzel-Larsen T, et al. Diabetes-Related emotional distress in adults: reliability and validity of the Norwegian versions of the problem areas in diabetes scale (paid) and the diabetes distress scale (DDS). Int J Nurs Stud 2012;49:174-82.

44 Polonsky WH, Fisher L, Earles J, et al. Assessing psychosocial distress in diabetes: development of the diabetes distress scale. Diabetes Care 2005;28:626-31.

45 Fisher L, Hessler DM, Polonsky WH, et al. When is diabetes distress clinically meaningful?: establishing cut points for the diabetes distress scale. Diabetes Care 2012;35:259-64.

46 Topp CW, Østergaard SD, Søndergaard S, et al. The WHO-5 well-being index: a systematic review of the literature. Psychother Psychosom 2015;84:167-76.

47 de Wit M, Pouwer F, Gemke RJBJ, et al. Validation of the WHO-5 well-being index in adolescents with type 1 diabetes. Diabetes Care 2007;30:2003-6.

48 Halliday JA, Hendrieckx C, Busija L, et al. Validation of the WHO-5 as a first-step screening instrument for depression in adults with diabetes: Results from Diabetes MILES - Australia. Diabetes Res Clin Pract 2017:132:27-35.

49 Hajos TRS, Pouwer F, Skovlund SE, et al. Psychometric and screening properties of the WHO-5 well-being index in adult outpatients with Type 1 or Type 2 diabetes mellitus. Diabet Med 2013;30:e63-9.

50 Rauwerda NL, Tovote KA, Peeters ACTM, et al. WHO-5 and BDI-II are acceptable screening instruments for depression in people with diabetes. Diabet Med 2018;35:1678-85.

51 Williams GC, McGregor HA, King D, et al. Variation in perceived competence, glycemic control, and patient satisfaction: relationship to autonomy support from physicians. Patient Educ Couns 2005;57:39-45.

52 STATA Data Analysis and Statistical Software.SE 16College Station, Texas, USA, 2020STATA. Available: https://www.stata.com/

53 Siddiqui OI. Methods for computing missing item response in psychometric scale construction. American Journal of Biostatistics 2015;5:1-6

54 Fayers P, Machin D. Missing data. In: Quality of life : the assessment, analysis and reporting of patient-reported outcomes. 3rd ed. Chichester, West Sussex: Wiley, 2016: 393-427.

55 Staniszewska S, Brett J, Simera I, et al. GRIPP2 reporting checklists: tools to improve reporting of patient and public involvement in research. BMJ 2017;358:j3453.
56 Mbuagbaw L, Kosa SD, Lawson DO, et al. The reporting of progression criteria in protocols of pilot trials designed to assess the feasibility of main trials is insufficient: a meta-epidemiological study. Pilot Feasibility Stud 2019;5:120.

57 Akl EA, Briel M, You JJ, et al. Potential impact on estimated treatment effects of information lost to follow-up in randomised controlled trials (LOST-IT): systematic review. BMJ 2012;344:e2809.

58 Brewster S, Bartholomew J, Holt RIG, et al. Non-attendance at diabetes outpatient appointments: a systematic review. Diabet Med 2020;37:1427-42.

59 Hynes L, Byrne M, Dinneen SF, et al. Barriers and facilitators associated with attendance at hospital diabetes clinics among young adults (15-30 years) with type 1 diabetes mellitus: a systematic review. Pediatr Diabetes 2016;17:509-18.

60 Norman GR, Sloan JA, Wyrwich KW. Interpretation of changes in health-related quality of life: the remarkable universality of half a standard deviation. Med Care 2003;41:582-92.

61 Skea ZC, Newlands R, Gillies K. Exploring non-retention in clinical trials: a meta-ethnographic synthesis of studies reporting participant reasons for drop out. BMJ Open 2019;9:e021959-e59.

62 Wilding S, Conner M, Sandberg T, et al. The question-behaviour effect: a theoretical and methodological review and meta-analysis. European Review of Social Psychology 2016;27:196-230.

63 Dennick K, Sturt J, Hessler D, et al. High rates of elevated diabetes distress in research populations: a systematic review and metaanalysis. Int Diabetes Nurs 2015;12:93-107.

64 Sturt J, Dennick K, Hessler D, et al. Effective interventions for reducing diabetes distress: systematic review and meta-analysis. Int Diabetes Nurs 2015;12:40-55.

65 Fisher L, Hessler D, Polonsky WH, et al. T1-REDEEM: a randomized controlled trial to reduce diabetes distress among adults with type 1 diabetes. Diabetes Care 2018;41:1862-9.

66 Hendrieckx C, Halliday J, Beeney L. Diabetes distress. In: Diabetes and emotional health: a practical guide for healthcare professionals supporting adults with type 1 and type 2 diabetes. 2nd ed. London: Diabetes UK, 2019: 53-70.

67 Griffiths F, Bryce C, Cave J, et al. Timely digital Patient-Clinician communication in specialist clinical services for young people: a mixed-methods study (the LYNC study). J Med Internet Res 2017;19:e102.

68 Moore GF, Audrey S, Barker M, et al. Process evaluation of complex interventions: medical Research Council guidance. BMJ 2015;350:h1258. 